\title{
A WALKING DISTURBANCE INDEX SUGGESTIONS FOR OPTIMIZED PATH SEARCH FOR THE PEOPLE WITH REDUCED MOBILITY
}

\author{
Mikyeong Moon, Yoonsik Bang, Kiyun Yu, Jiyoung Kim* \\ Seoul Nat'1 University, Civil Environmental Engineering, Master's Student (coco0320@ snu.ac.kr) \\ Seoul Nat'l University, Civil Environmental Engineering, Doctoral Student (bangys1004@snu.ac.kr) \\ Seoul Nat'l University, Civil Environmental Engineering, Professor (kiyun@ snu.ac.kr) \\ Seoul Nat'1 University, Institute of Construction and Environmental Engineering, Research Professor (soodaq@ snu.ac.kr)
}

KEY WORDS: AHP, FUZZY LOGIC, OPTIMIZED PATH FOR THE PEOPLE WITH REDUCED MOBILITY

\begin{abstract}
:
Recently, due to the increased penetration of smart devices and the development of geographic information system (GIS) technology, various route guidance services for pedestrians have been developed. However, until now, pedestrian navigation services for the people with reduced mobility (people who experience discomfort in transportation) including wheelchair users, the elderly, and pregnant women have not been provided. In this study, we present a walking disturbance index methodology for searching an optimized path for the people with reduced mobility by defining the factors that affect the walking of the people with reduced mobility and deriving the weights of these factors. In future research, we expect to be able to provide a navigation system that gives an optimized path for the people with reduced mobility using this method.
\end{abstract}

\section{INTRODUCTION}

According to the UN Human Rights Charter, enacted in 1948, Move right is one of the basic human rights. However, vulnerable users have mobility constraints due to a physical disorder and are therefore often alienated socially, economically, and culturally. In particular, they face more problems when they are in an unfamiliar environment. Indeed, many factors hinder the walking ability of the people with reduced mobility such as narrow road width, steep slopes, and uncomfortable road surface. We therefore need to develop technologies that help the movement of the people with reduced mobility.

Supported by the development of GIS technology and the increased penetration of smart devices, a wide range of route guidance services are currently being provided at a rapid rate, not only for vehicle use but also for pedestrians. However, people with reduced mobility people are more affected by physical and environmental parameters when they are walking than are general pedestrians. Therefore, we need to provide a route guidance service that considers this phenomenon. Conventional route guidance services for vehicles and general pedestrians do not take into account the parameters recognized as sidewalk obstacles such as steep slopes, narrow road width, and steps. Also, the physical and cognitive characteristics of the types of the people with reduced mobility (wheelchair users, pregnant women, strollers, the elderly, children, etc.) differ from each other. Therefore, the elements that must be considered by the people with reduced mobility when choosing a path differ from those considered by able persons. Therefore, we need to provide a route guidance service that considers the elements that impede the walking of the people with reduced mobility.

In this study, we define the parameters that impede walking to each type of people with reduced mobility and propose an optimized path search algorithm for the people with reduced mobility that merges with distance by quantifying the degree of disturbance of the abovementioned parameters.

\section{OPTIMIZED PATH SEARCH ALGORITHM FOR THE PEOPLE WITH REDUCED MOBILITY}

Figure 1 shows the walking disturbance index for searching an optimized path for the people with reduced mobility and the path search algorithm using a walking disturbance index.

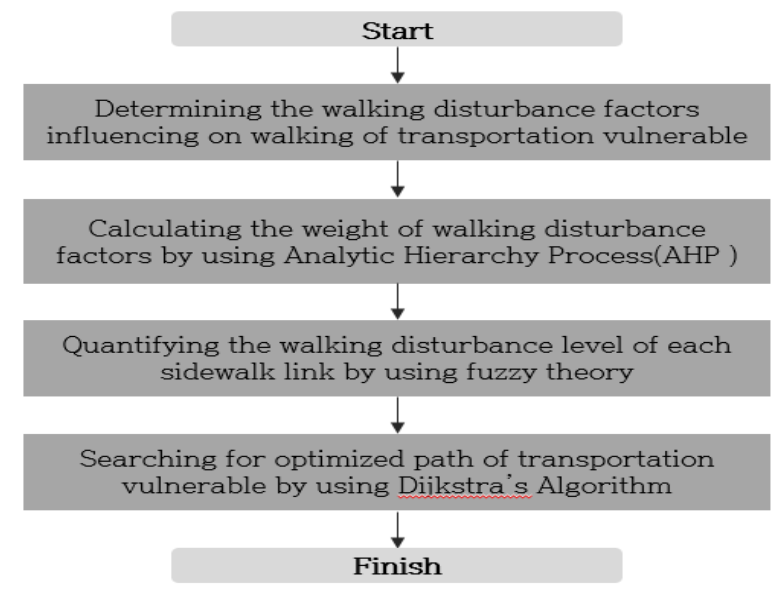

Figure 1. Flowchart of process of searching for optimized path

\footnotetext{
* Corresponding author
} 
The Walking Disturbance Factors are defined by selecting the factors that affect the walking ability of the people with reduced mobility. The weights of the defined Walking Disturbance Factors are calculated by using an Analytic Hierarchy Process (AHP) (Satty, 1980) and the Walking Disturbance Level is derived through the fuzzy inference system in which the inputs are the attributes of each link and the weight of each factor. The walking disturbance index is then calculated using the formula (Figure 2) considering the length of link segment. An optimized path for the people with reduced mobility with a minimized total walking disturbance index is then searched.

\subsection{Determining the Walking Disturbance Factors influencing the walking ability of people with reduced mobility.}

Due to their physical constraints, more obstacles affect the walking ability of the people with reduced mobility than affect general pedestrians. Curb height, steps, and steep slopes are examples of walking obstacles. First, by analyzing these obstacles, we need to extract the parameters that influence the walking ability of the people with reduced mobility in order to search for the optimum path. The variables considered in previous studies (Osok Ji, 2008) which deal with the walking environmental indicators are primary candidate parameters used to obtain the reliability of the Walking Disturbance Factor. The factors that can be used in GIS analysis are defined as the final Walking Disturbance Factors to be applied to the path search algorithm. The final Walking Disturbance Factors are steep slope, narrow sidewalk width, steps, poor sidewalk surface, total route length, whether there are walking trails, and walking obstacles (cracks, inhibiting entry rods etc.).

2.2 Calculating the weights of Walking Disturbance Factors by using Analytic Hierarchy Process (AHP).

The people with reduced mobility can be classified into the people with reduced mobility in a broad sense and the people with reduced mobility in a narrow sense. In this study, the people with reduced mobility in the narrow sense include those individuals who have limited movement ability because of their physical situation, such as wheelchair users, the elderly, pregnant women, and pedestrians with crutches.

Even though the same walking disturbance index is used, the degree of importance of walking for the people with reduced mobility differs because physical disability and cognitive characteristics differ according to the type of people with reduced mobility. Therefore, the weights of the Walking Disturbance Factors need to be calculated to represent the type of people with reduced mobility. In this study, the Analytic Hierarchy Process (AHP) which analyzes the qualitative elements quantitatively based on an expert survey is used to calculate the weight.

The relative importance of the Walking Disturbance Factors is derived through a survey to compare each pair of Walking Disturbance Factors. Experts' preferences are represented by semantic expression and are invested with an appropriate number (1 9 points), where 1 implies equality and 9 implies the factor is considerably important in the degree of walking disturbance for the people with reduced mobility. Using the values obtained through the pair comparison, we estimate the relative weights of the Walking Disturbance Factors and ensure the reliability of the weights by deriving the consistency index.

\subsection{Quantifying the Walking Disturbance Level of each sidewalk link by using fuzzy theory.}

The Walking Disturbance Level of each sidewalk link is quantified based on the weights of the Walking Disturbance Factors. The Walking Disturbance Level is a value from 0 to 5, which means the extent to which walking is hindered and it is calculated by integrating the attribute of each link and the weight obtained in the previous step. This step uses the fuzzy theory (Zadeh, 1965) for flexible and precise decision-making by acknowledging the ambiguity of the analysis criteria and quantifying the extent of the importance of each factor.

The fuzzy inference system is implemented by defining the membership function for each Walking Disturbance Factor and fuzzy rule. The inputs of the system are the weights derived in the previous step and the membership function value utilizing the properties of each sidewalk link. The outputs of the system are the Walking Disturbance Levels represented by a number between 0 and 5 points. Examples of the fuzzy rules proposed in this study are as follows.

Rule 1 : If the sidewalk width is narrow and the weight of the sidewalk width factor is high, then the Walking Disturbance Level is Very Uncomfortable.

Rule 2 : If the slope of the sidewalk is very steep and the weight of the slope factor is low, then the Walking Disturbance Level is Uncomfortable.

In this process, the fuzzy inference system which is implemented on the basis of the above-described fuzzy rules shows the various Walking Disturbance Levels for each type of people with reduced mobility person.

\subsection{Searching for optimized path of people with reduced mobility by using Dijkstra's Algorithm.}

Finally, the Walking Disturbance Index considers the Walking Disturbance Level, and the Length factor is then calculated. The optimized path for the people with reduced mobility is searched by using Dijkstra's Algorithm (Dijkstra, 1959), which is one of the shortest path algorithms. The formula to determine the Walking Disturbance Index is shown in Figure 2.

\section{Walking Disturbance Index = Walking Disturbonce Level $x$ Length $\times$ Length Weight}

Figure 2. Walking Disturbance Index Formula

The Dijkstra's Algorithm is used to give an optimized path considered Walking Disturbance Factors and length of path for people with reduced mobility by searching a path which is minimized the Walking Disturbance Index. In this step, the Walking Disturbance Index is set to cost for path searching. 


\section{CONCLUSION}

In this study, we define the factors affecting the walking ability of the people with reduced mobility and derive the weight for each Walking Disturbance Factor by using the AHP technique. Moreover, by using the weight derived in the previous step, the Walking Disturbance Level is calculated based on the fuzzy theory and an algorithm is proposed to find the path that will minimize the Walking Disturbance Index. We expect that this study will provide a base for the development of a navigation system for the people with reduced mobility. In future research, we plan to develop the navigation for people with reduced mobility which provide the optimize route applied the proposed algorithm.

\section{ACKNOWLEDGEMENT}

This work was supported by the ICT R\&D program of MSIP/IITP. [B0101-15-1349, Development of Volunteered Geospatial Information Platform Technology and Application for the Elderly and Disabled]. This research was supported by a grant (15CHUD-C061156-05) from National Spatial Information Research Program funded by Ministry of Land, Infrastructure and Transport of Korean government.

\section{REFERENCES}

Dijkstra, E.W., 1959. A note on two problems in connexion with graphs. Numerische Mathematik, 1, 271.

Osok Ji, 2008. A study on Satisfaction for Pedestrian Environment, Gyeonggi Research Institute.

Satty, T.L., 1980. The analytic hierarchy process. New York: McGraw-Hill.

Zadeh, L.A., 1965. Fuzzy sets. Information and Control, 8, 338353. 